\title{
Video Article \\ Microinjection of Zebrafish Embryos to Analyze Gene Function
}

\author{
Jonathan N. Rosen ${ }^{1,2}$, Michael F. Sweeney ${ }^{1}$, John D. Mably ${ }^{1,2}$ \\ ${ }^{1}$ Department of Genetics, Harvard Medical School \\ ${ }^{2}$ Department of Cardiology, Children's Hospital Boston
}

Correspondence to: John D. Mably at john.mably@childrens.harvard.edu

URL: https://www.jove.com/video/1115

DOI: doi:10.3791/1115

Keywords: Developmental Biology, Issue 25, zebrafish, morpholino, development, microinjection, heart of glass, heg

Date Published: $3 / 9 / 2009$

Citation: Rosen, J.N., Sweeney, M.F., Mably, J.D. Microinjection of Zebrafish Embryos to Analyze Gene Function. J. Vis. Exp. (25), e1115, doi:10.3791/1115 (2009).

\section{Abstract}

One of the advantages of studying zebrafish is the ease and speed of manipulating protein levels in the embryo. Morpholinos, which are synthetic oligonucleotides with antisense complementarity to target RNAs, can be added to the embryo to reduce the expression of a particular gene product. Conversely, processed mRNA can be added to the embryo to increase levels of a gene product. The vehicle for adding either mRNA or morpholino to an embryo is microinjection. Microinjection is efficient and rapid, allowing for the injection of hundreds of embryos per hour. This video shows all the steps involved in microinjection. Briefly, eggs are collected immediately after being laid and lined up against a microscope slide in a Petri dish. Next, a fine-tipped needle loaded with injection material is connected to a microinjector and an air source, and the microinjector controls are adjusted to produce a desirable injection volume. Finally, the needle is plunged into the embryo's yolk and the morpholino or mRNA is expelled.

\section{Video Link}

The video component of this article can be found at https://www.jove.com/video/1115/

Protocol

\section{Part 1: Egg production and collection}

1. The night prior to injection, set up the fish in breeding tanks with dividers in place. To increase total egg production, fish can be set up in a ratio of two females to one male if desired.

2. The following morning, after the room lights turn on, pull the dividers from several tanks and allow for approximately 20 minutes of undisturbed mating time.

3. Using a strainer, collect the eggs from the breeding cages and rinse them with egg water. Pour eggs into a Petri dish with egg water and remove unfertilized eggs and debris with a transfer pipette. Fish can be regrouped in larger tanks to produce additional rounds of eggs for injection. Adjust the timing of egg collection to allow for maximum numbers of eggs to be produced without letting them pass the single cell stage.

4. Place a microscope slide in the inverted lid of a $100 \mathrm{~mm}$ Petri dish. Use a transfer pipette to line up the eggs against the side of the slide forming a single column. Remove excess egg water from the slide by pressing a Kimwipe against the side opposite the eggs. (Figure $1 \mathrm{~A}$ )

\section{Part 2: Needle pulling, loading, and preparation}

1. With a micropipette puller, pull a $1.0 \mathrm{~mm}$ OD glass capillary into two needles and store in a $150 \mathrm{~mm}$ Petri dish by laying over silly putty ramps. Needles can be pulled in advance.

2. Backload the needle with $3 \mu \mathrm{L}$ of injection material using a microloader pipette. Shake the bolus toward the needle tip until there are few or no bubbles remaining.

3. Turn on the air source and microinjector. Insert the needle into the microinjector and insure a tight seal within the housing. Check that the micromanipulator is in a proper position to allow for a wide range of movement and adjustment. Bring the needle tip into the plane of view of the microscope, high off the stage, and focus on the thinnest region of the tip. Use a pair of sharp forceps to pinch off the needle at a point which leaves the needle narrow enough to pierce the chorion and yolk but still capable of delivering a consistent bead size. A drop of mineral oil on a micrometer can be used to calculate the volume of each injection. When injected into the oil, a bead with a diameter of $0.1 \mathrm{~mm}$ contains $500 \mathrm{pL}$ of injection material (figure $1 \mathrm{~B}$ ); injection volumes of $500 \mathrm{pL}$ or $1 \mathrm{~nL}$ are typically used. Depress the foot pedal and monitor the size of the bead while trimming the needle and adjusting the injection pressure as needed. Ideal injection volumes will fill approximately $10 \%$ of the egg volume. The quality of the needle tip is crucial to both the ease of injection and the quality and consistency of the results. 


\section{Part 3: Injection}

1. Ensure the embryos have not developed past the four-cell stage. Ideally, embryos should be at the one-cell stage.

2. Lower the needle toward the column of eggs, holding the dish in place with your opposite hand.

3. Pierce the surface of the chorion and enter the yolk in one smooth stroke while watching for any crushing or tearing of the yolk sac. Inject the injection material into the yolk (figure 1C). Avoid injecting air bubbles or stretching the yolk as either can be lethal to the embryo. Working down the line, adjust the pressure as needed to maintain a consistent bead size and, using a pipette tip, remove eggs which look unfertilized or are destroyed during the injection process.

4. After completing a column of eggs, use a gentle stream of egg water to move the injected eggs into a clean Petri dish. Repeat as necessary. Keep several uninjected embryos as a control. At the end of day one, remove dead embryos and record the number of injected embryos. Replace the egg water in the dish periodically to reduce the chance of infection.

\section{Part 4: Representative results}

Depending on what is being injected, embryos may survive at a lower rate than their uninjected siblings. Fortunately, it is easy to inject huge numbers of them, so this is rarely a problem. It is normal for morphants to exhibit slightly delayed development.

To illustrate the results of microinjection, we used this protocol to manipulate levels of the protein Heart of Glass (Heg). Heg is a transmembrane protein required for normal heart development. In its absence, embryos develop hearts with giant inflow tracts and chambers ${ }^{1}$. We injected two previously undescribed morpholinos against heg, heg_e3i3_egfr1 and heg_e4i4_egfr2, at a concentration of $500 \mu \mathrm{M}$. At 2 days post fertilization, the uninjected sibling controls appear normal, as expected (figure $2 \mathrm{~A}$ and $\mathrm{B}$ ). Embryos injected with heg_e3i3_egfr1 have brain edema (figure 2C). Although the hearts of most of these morphants have altered morphology, their chambers are normally sized (figure 2D). Embryos injected with heg_e4i4_egfr2 exhibit variable heart defects. Some appear normal, while others have large inflow tracts and moderately enlarged atria (figure $2 \bar{E}$ and $\bar{F}$ ). The heg mutant, as previously reported ${ }^{1}$, has a hugely enlarged inflow tract and atrium (figure $2 \mathrm{G}$ and $\mathrm{H}$ ).

We also injected wildtype embryos with $400 \mathrm{ng} / \mu \mathrm{L}$ mRNA transcribed from full-length heg cDNA. While uninjected controls develop normally (figure $3 \mathrm{~A}$ and $\mathrm{B}$ ), embryos injected with heg mRNA exhibit a spectrum of phenotypes ranging from wildtype appearance to severe cyclopia, shortening of the main body axis, necrosis of the head and body, and midline defects (figure $3 \mathrm{C}$ and $\mathrm{D}$ ).

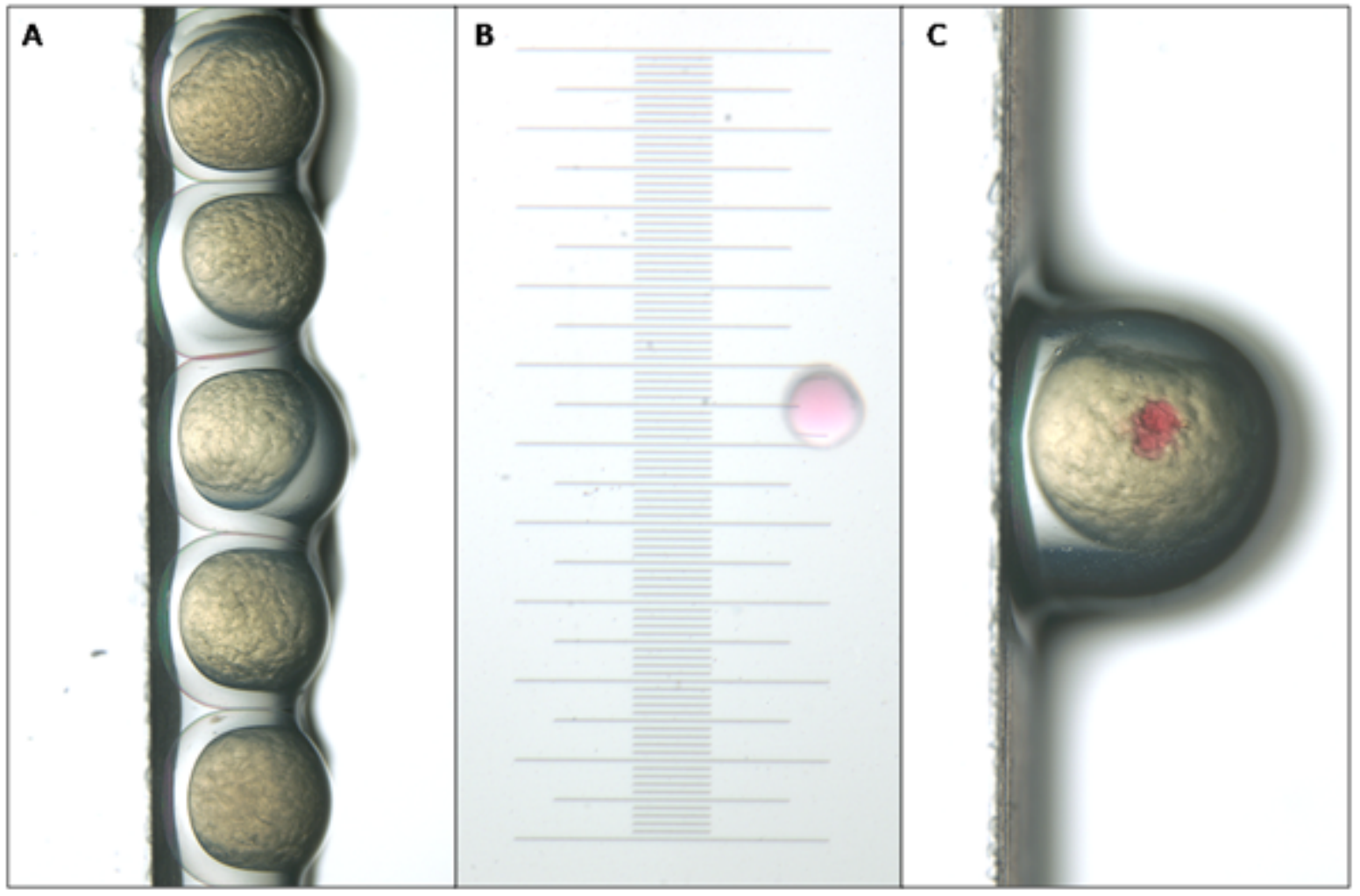

Figure 1. Embryos to be injected are lined up against a microscope slide in a Petri dish (A). Injection volume is determined by injecting into mineral oil placed on a micrometer. An injection volume of $500 \mathrm{pL}$, which is commonly used, has a diameter of $0.1 \mathrm{~mm}(\mathrm{~B})$. Immediately after injection, the morpholino or mRNA is visible as a punctuate spot in the yolk (C). 


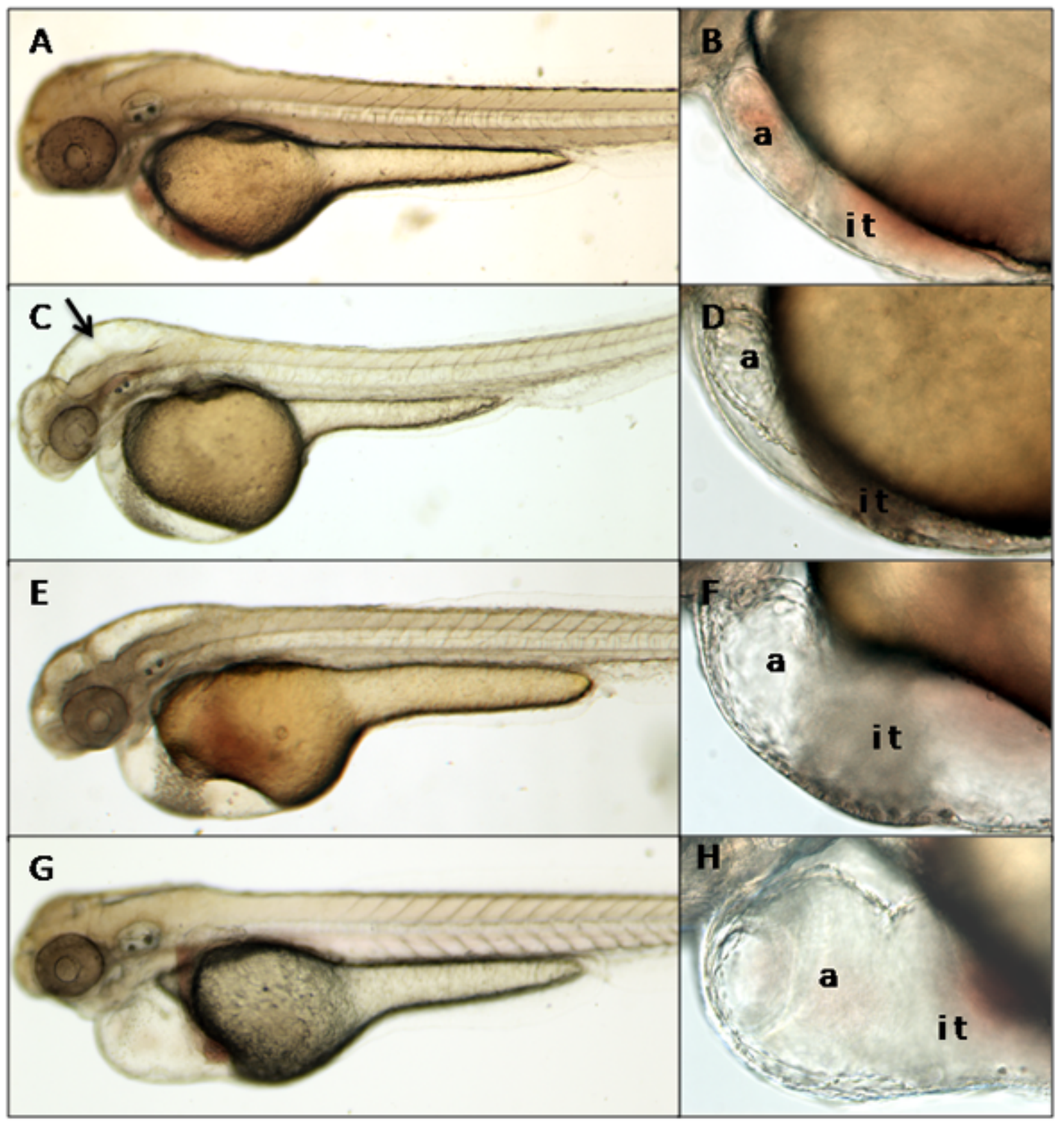

Figure 2. At 2 days post fertilization, uninjected embryos have no gross defects (A) or heart phenotype (B). Embryos injected with the morpholino heg_e3i3_egfr1 have brain edema (C, arrow) but normally sized heart chambers (D). Some embryos injected with the morpholino heg_e4i4_egfr2 have enlarged inflow tracts and atria $(E, F)$. heg mutants have severely enlarged inflow tracts and atria $(G, H)$. (A), $(C),(E)$, and $(\mathrm{G})$ are $4 \times \mathrm{DIC}$ images; $(\mathrm{B}),(\mathrm{D}),(\mathrm{F})$ and $(\mathrm{H})$ are $20 \mathrm{x}$ DIC images. a=atrium, i t= inflow tract. 


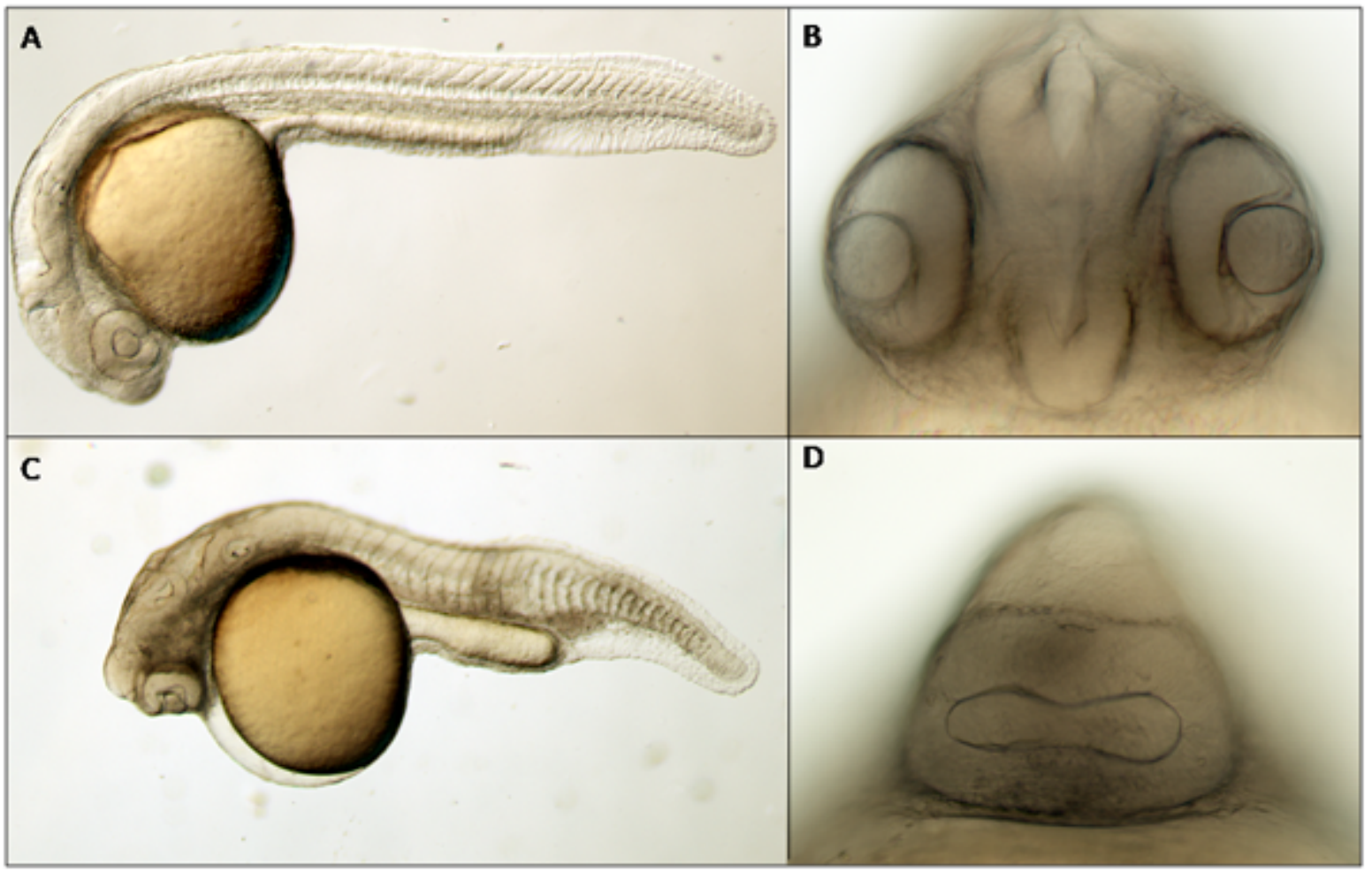

Figure 3. At 24 hours post fertilization, uninjected embryos have a long, straight body with no necrosis or midline defects (A) and two clearly defined eyes with a neural tube between them (B). Some embryos injected with heg mRNA, in contrast, exhibit a shortened body axis, necrosis, misshaped somites (C), and cyclopia (D). (A) and (C) are 4x DIC images; (B) and (D) are 20x DIC images.

\section{Discussion}

One of the strengths of the zebrafish model system is the ease with which specific gene products can be added to or eliminated from the embryo by microinjection. To ubiquitously overexpress a particular protein, the mRNA encoding it is injected into the yolk at the 1-cell stage. During the embryo's subsequent development, the RNA is distributed throughout the organism and translated. Conversely, to eliminate a particular protein, morpholinos are used. Morpholinos are synthetic oligonucleotides designed with antisense complementarity to specific RNAs. Like mRNAs, morpholinos are injected into the yolk at the 1-cell stage. Inside the embryo they bind their target RNAs, preventing translation.

The main modification that needs to be made for each morpholino or mRNA is the concentration of injected material. Too high a concentration of either morpholino or mRNA may cause non-specific toxicity; while each morpholino must be tested empirically to determine its optimal concentration, typically morpholino concentrations between $200 \mu \mathrm{M}$ and $500 \mu \mathrm{M}$ knock down gene activity effectively without causing nonspecific defects. The precise size of the needle opening is not crucial. Within a range of needle sizes, injection pressure and injection time can be adjusted to produce a bolus of the correct volume.

Morpholinos have many applications, including the functional dissection of domains within a protein. When designed to target specific exonintron junctions, morpholinos will prevent splicing from occurring there. We examined the effects of two morpholinos that bind exon-intron junctions in the pre-mRNA of heg. The morpholino heg_e3i3_egfr1 binds the junction between exon 3 and intron 3, preventing the splicing machinery from incorporating exon 3 into mature transcripts. The morpholino heg e4i4 egfr2 similarly removes exon 4 . Both exons 3 and 4 encode domains containing EGF-like repeats. Some embryos injected with heg_e4i4_egfr2 moderately phenocopy the mutant; further experiments will be required to understand the role of Heg exon 4 in heart development. Surprisingly, embryos injected with heg_e3i3_egfr1 have brain edema, a feature not seen in heg mutants. This may be due to the ability of morpholinos to bind maternal transcripts that would be unaffected in zygotic mutants.

\section{Acknowledgements}

We acknowledge members of the Mably lab and Narie Storer for their technical assistance, and the American Heart Association (NCRP Scientist Development Grant 0635363N) and the National Heart, Lung, and Blood Institute (Grant SCCOR RFA HL02-027) for funding. 


\section{References}

1. Mably, J.D., Mohideen, M.P.K., Burns, C.G., Chen, J., \& Fishman, M.C. heart of glass regulates the concentric growth of the heart in zebrafish. Curr. Biol. 13, 2138-2147, (2003). 